\title{
Prediction of bed-leaving behaviors using piezoelectric non-restraining sensors
}

\author{
H. Madokoro, N. Shimoi, and K. Sato \\ Faculty of Systems Science and Technology, Akita Prefectural University, 84-4 Tsuchiya Aza Ebinokuchi, \\ Yurihonjo City, Akita, 015-0055, Japan \\ Correspondence to: H. Madokoro (madokoro@ akita-pu.ac.jp)
}

Received: 27 December 2012 - Revised: 26 March 2013 - Accepted: 14 April 2013 - Published: 30 April 2013

\begin{abstract}
This paper presents a sensor system to predict behavior patterns that occur when patients leave their beds. We originally developed plate-shaped sensors using piezoelectric elements. Existing sensors such as clip sensors and mat sensors require restraint of patients. Moreover, these sensors present privacy problems. The features of our sensors are that they require no power supply or patient restraint. We evaluated our system using a basic experiment to predict seven behavior patterns. We obtained a result of predicted behavior patterns related to bed-leaving using only six sensors installed under a bed. Especially, our system can correctly detect behavior patterns of lateral sitting, which is a position that occurs when a patient tries to leave from the bed, and terminal sitting, which is the position immediately before bed-leaving. They were discerned from other behavior patterns.
\end{abstract}

\section{Introduction}

According to the National Population Census 2010 in Japan, the aging rate of the country is $23.1 \%$ (NPC, 2010). This rate implies that Japan has entered a super-aging society, which is defined as the rate of the number of people aged over 65 $\mathrm{yr}$ old is greater than 20 percent of the total population. A report of National Institute of Population and Social Security Research estimated that a quarter of Japanese residents will be more than 65 years old in 2015 (IPSS, 2012). Along with the longevity of the society, labor shortages will become severe, especially at nursing-care facilities (Knokuchi, 2008). Few caretakers must care for numerous patients. For this situation, caretakers monitor patients inadequately, especially during sleep at night (Yamada et al., 2010). One approach to this problem is to use bed-leaving sensors that signal when patients leave from their beds. They can be used to prevent falling from their beds. The number of hospitals and nursingcare facilities using these sensors has increased (Imaizumi et al., 2010; Matsuda et al., 2003). Table 1 presents existing sensors to be used actually. We compare features of these sensors related to cost, detection speed, accuracy, privacy, and restrictiveness.
Actually, clip sensors are the lowest-cost sensors that can be introduced easily. This is a simple sensor attached to a patient's clothing (Tatsumi et al., 2007). According to protection of human rights, the usage of clip sensors has been prevented recently because it requires constraint of the wearer. For the performance of clip sensors, malfunctions and anomaly detections occur frequently because of the binary response, ON or OFF, used to detect bed-leaving behaviors. Regarding the reliability and perspective of management, clip sensors are insufficient to prevent falling from a bed completely. Moreover, accidents caused by binding of the neck in a cable have been reported (Tatsumi et al., 2007). We regard clip sensors as inadequate for use at clinical sites, although it is easy to introduce them at low cost.

Recently, mat sensors are widely used as a low-cost and convenient sensors that can be installed easily (Kondo et al., 2006). Haruyama et al. developed an alarm system to detect patients leaving from their beds using mat sensors (Haruyama et al., 2006). Medical and welfare suppliers released various mat sensors installed on a floor, a bed, or on rolling handrails. Mat sensors used on a floor are unnecessary for authentication for medical devices under the pharmaceutical law. Regarding the performance of detection, a problem 
Table 1. Comparison of characteristics of existing bed-leaving sensors.

\begin{tabular}{lllllll}
\hline Sensor type & Cost & Timing & Accuracy & Privacy & Restriction & Reference \\
\hline Clip & Low (up to \$100) & Fast & Low & High & Yes & Tatsumi et al. (2007) \\
Bed mat & Low (up to \$200) & Fast & Low (70.1\%) & Low & No & Kondo et al. (2006) \\
Floor mat & Low (up to \$200) & Slow (after standing) & Low & Low & No & Haruyama et al. (2006) \\
Handrail & Low (up to \$300) & Fast & Low & Low & No & Haruyama et al. (2006) \\
Camera & High & Fast & High & High & No & Seki et al. (2002) \\
Infrared & High & Fast & High & High & No & Hirasawa et al. (2008) \\
Supersonic & High & Fast & High (95.1\%) & High & No & Shimizu et al. (2009) \\
Strain gauge & High & Fast & High $(99.0 \%)$ & Low & No & Uezono et al. (2010) \\
\hline
\end{tabular}

of a delay remains because of the response after sitting at the end of the bed, although such sensors are easy to produce and to sell. Moreover, sensor responses are apparent when medical staff members such as a nurse or a medical doctor walk on the mat. To distinguish the responses of patients and medical personnel is a challenging problem for signal pattern recognition. Sensors rolled over handrails not only obstruct a view of a bed, but also present a risk of removal of a sensor when a patient finds it and feels negatively about being restrained. Furthermore, false detection occurs when patients leave their bed without gripping a handrail. Mat sensors installed on a bed can detect bed-leaving with higher reliability than other mat sensors. However, existing mat sensors are actuated by a binary response similar to that for clip sensors. Early detection is not realized, especially in the initial stage of bed-leaving behavior.

Large-scale systems using numerous sensors of various types have been proposed for prediction at the initial stage to measure behavior patterns in detail. Shimizu et al. proposed a bed-leaving detection system using ultrasonic array sensors (Shimizu et al., 2009). They evaluated their system at a hospital as a demonstration experiment. Hirasawa et al. proposed a method to expose infrared rays to the upper part of the bed as a system to prevent falling accidents (Hirasawa et al., 2008). Uezono et al. proposed a large-scale monitoring system for detecting bed-leaving behavior patterns using 96 strain gauges assigned for a reticular pattern (Uezono et al., 2010). These large-scale sensor systems can realize higher accuracy and more stable detection than low-cost sensors, such as clip sensors or mat sensors, can. However, these sensors are not put into practical use because of their cost. Moreover, high expenditures are necessary to replace a bed or for construction for installation whenever these systems are improved for practical use in a market.

Using a camera as a bed monitoring sensor can provide a low-cost system. Moreover, it can obtain much information for a subject. However, it is a challenging task to predict behavior patterns obtained from images, even when stateof-the-art computer vision technologies are used. For this method, medical staff members must observe images di- rectly. It is impossible to monitor numerous subjects simultaneously with a few operators. Moreover, we must consider aspects of human rights and quality of life (QOL). Especially, it is impossible to recognize behavior patterns related to bed-leaving using only sensor responses, even when detailed analyses are conducted, because behavior patterns differ among people (Seki et al., 2002). Moreover, monitoring using a camera imposes a mental load on patients because they feel as though they are under surveillance all day and all night.

For solving these problems, this paper presents an nonrestraining sensor system using piezoelectric films. We aim at reducing the amount of data used for predicting and minimizing incorrect recognition given the minimum number of sensors employed. Our sensor requires no electric power for detection because it uses piezoelectric elements. Moreover, we developed an integrated system to send data obtained from sensors between a capturing device and a monitoring terminal computer using a close-range wireless module. We developed a microcomputer board in the device to remove noise from sensor data. We evaluated our proposed system at an environment that represents a clinical site. We obtained a result to determine and to predict seven behavior patterns related to bed-leaving. Moreover, we obtained a decision rule of bed-leaving from histograms for actual application to a clinical site.

\section{Proposed sensor system}

\subsection{Sensor}

High-performance and functional sensors of various types were used for existing bedside monitoring systems for targeting expensive care or medical treatments (Haruyama et al., 2006; Shimizu et al., 2009; Hirasawa et al., 2008; Uezono et al., 2010). In contrast, we designed a system providing low cost and user-friendliness for practical use. Our originally developed plate-shaped sensors can be installed easily under a bedsheet. 


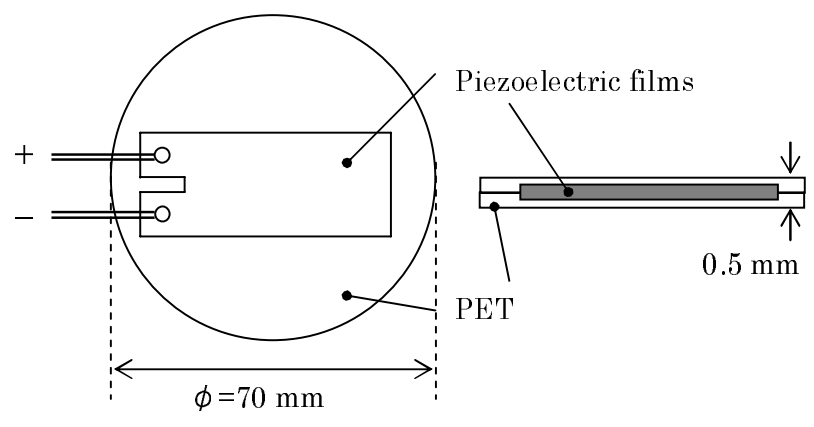

Figure 1. Design structure of prototype sensor.

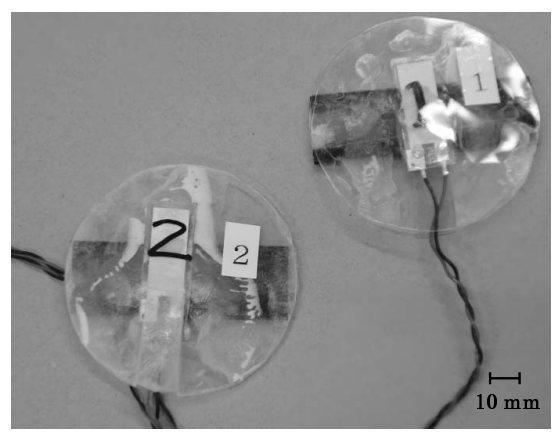

Figure 2. Overview photograph of prototype sensor.

As a prototype, we developed a sensor using piezoelectric film DT2-028K/L by Measurement Specialties Inc. Table 2 presents specifications of the DT2-028K/L film (Measurement Specialties Inc., 2009). We fixed a piezoelectric film between two polyethylene terephthalate (PET) plates of laminated polyester. The polyester and PET plate sizes were, respectively, $125 \mu \mathrm{m}$ and $\phi 70 \times 0.5 \mathrm{~mm}$. Figures 1 and 2 respectively depict the design structure and an overview photo of our prototype sensors.

Output voltage is generated from the bent piezoelectric films when a subject transfers body weight on the bed. This sensor can measure recursively because the reference potential is offset when the bending stops. Moreover, the strength of weight according to changes of the body is obtainable linearly because the bend of the piezoelectric film and output voltage has a relation of proportionality. Furthermore, piezoelectric films are less troublesome and provide no false operations because they have simple wiring without electric power supply for measurements. Additionally, we can provide a low-cost system requiring no maintenance related to replacement of a battery.

\subsection{System structure}

Figure 3 portrays the entire structure of our sensor system that we originally developed for this study. Our system comprises six plate-shaped sensors installed on the bed and a microprocessor board that can obtain output voltage from each
Table 2. Specifications of Piezoelectric film DT2-028K/L (Measurement Specialties Inc.).

\begin{tabular}{ll}
\hline Parameter & Value \\
\hline Minimum impedance & $1.0 \times 10^{3} \Omega$ \\
Output voltage & $10 \mathrm{mV}-100 \mathrm{~V}$ \\
Stress constant $\left(\mathrm{g}_{31}\right)$ & $216 \mathrm{mV} \mathrm{m} \mathrm{s}$ \\
Capacity & $380 \mathrm{pF} \mathrm{cm}^{-2}$ \\
Young's modulus & $2-4 \times 10^{6} \mathrm{Nm}^{2}$ \\
Voltage resistance & $80 \mathrm{~V} \mathrm{~m}^{-1}$ \\
Tension strength $\left(T_{\mathrm{B}}\right)$ & $140-210 \times 10^{3} \mathrm{~N} \mathrm{~m}^{-2}$ \\
Tension strength $\left(T_{\mathrm{Y}}\right)$ & $30-55 \times 10^{3} \mathrm{~N} \mathrm{~m}^{-2}$ \\
stretch strength $\left(S_{\mathrm{B}}\right)$ & $2.5-4.0 \%$ \\
stretch strength $\left(S_{\mathrm{Y}}\right)$ & $2.0-5.0 \%$ \\
Operating temperature & $0-70{ }^{\circ} \mathrm{C}$ \\
\hline
\end{tabular}

Partition

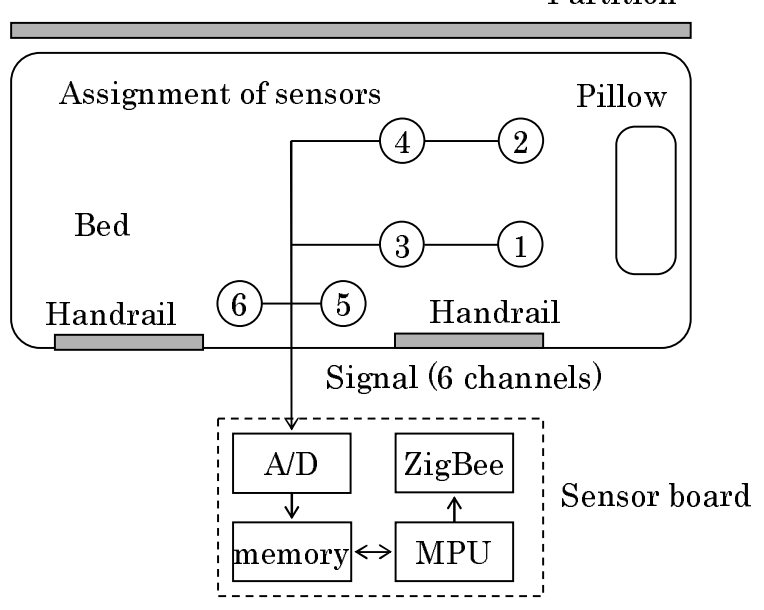

Figure 3. Block diagram of our system and assignment of sensors on a bed.

sensor for sending to a monitoring terminal computer. The output voltage is generated from these sensors when piezoelectric films are bent to receive body weight changes with movements of a subject caused by rolling or rising on a bed. The assignment of six sensors is $\mathrm{S} 1$ and $\mathrm{S} 2$ for the shoulder part, S3 and S4 for hip part, and S5 and S6 for the terminal part. We assigned these six sensors referring to the literature related to the development of a monitoring system for users of welfare care beds (Imaizumi et al., 2010; Matsuda et al., 2003).

For this assignment, a subject is available on the bed if responses from S1, S2, S3, and S4 are given alternately. In this case, the system can recognize that a subject is sleeping or rolling on the bed. In contrast, the system can detect a subject attempting to try to leave from the bed when responses are given from S5 or S6. Moreover, the system judges that a subject left from the bed completely if no sensor gives any response. This is the boundary to determine complete leaving or estimated leaving. Moreover, it can be estimated that a 


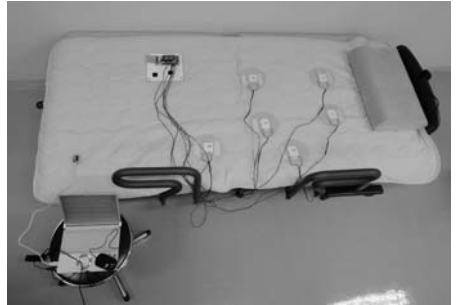

(a) Whole system

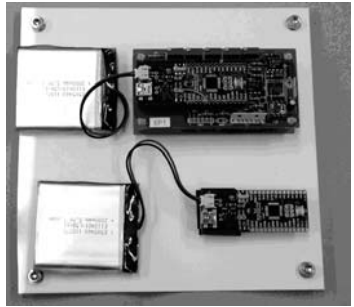

(b) Sensor boards
Figure 4. Photographs of our experimental environment, microprocessor board, and monitoring tool.

subject becomes unconscious if no sensors has any response. Both are severe situations. We consider that our system can provide attention to a nurse or a medical doctor if our system is combined with a hospital information network system such as a nurse call network.

The primary feature of our system is that it realizes monitoring using non-restraining sensors. We consider QOL for a patient to live life normally. Our system requires no supervision using infrared cameras or constraining sensors such as clip sensors. Moreover, we can create a low-cost system using piezoelectric films as sensors that can function with little trouble or missed operations, and with remarkable characteristics for pressure resistance.

Figure $4 \mathrm{~b}$ depicts the exterior of our capturing board equipped with a microprocessor and a wireless module. This board obtains output voltage from each sensor for wireless communication. For this study, we developed this board using Open Source Hardware ArduinoFIO. With consideration of power consumption, we used short-range wireless communication standard ZigBee communicated with a monitoring terminal computer. The input of this board is four channels. We used two boards for six sensors.

We set thresholds for measurement values from each sensor to reduce the communication traffic and the total amount of useless data for bed-leaving prediction. Moreover, we developed a function to analyze monitoring data as behavior logs. We originally developed software to recognize positions or movements of a subject from output patterns in each sensor used for monitoring by a terminal computer.

\subsection{Detection and recognition algorithms}

The target behaviors for recognition or prediction of bedleaving consist of three patterns: sleeping, attempted leaving, and complete leaving. For this study, we also attempt to classify detailed behavior patterns from the responses of six sensors. The behavior of sleeping consists of three patterns: upside sleeping, left-side sleeping, and right-side sleeping. The behaviors of attempted leaving consist of three patterns (Mogi et al., 2011). The first behavior pattern is the status of sitting on the bed to the longitudinal side. For this position,

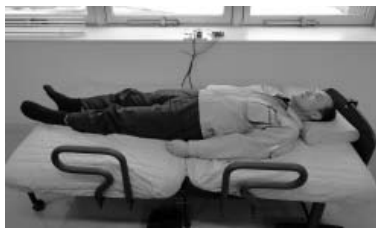

(1) Normal sleeping

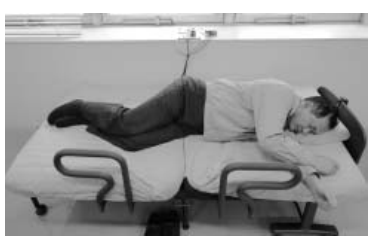

(3) Rolled left side

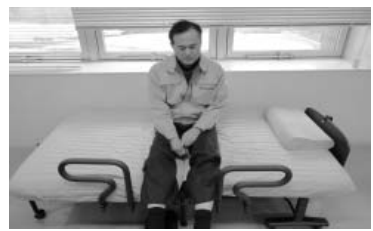

(5) Lateral sitting

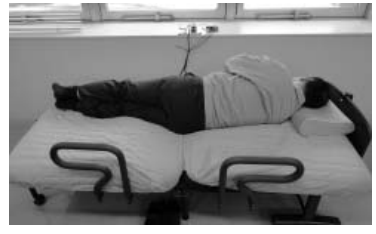

(2) Rolled right side

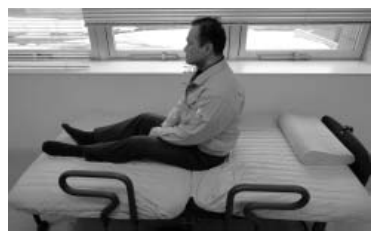

(4) Longitudinal sitting

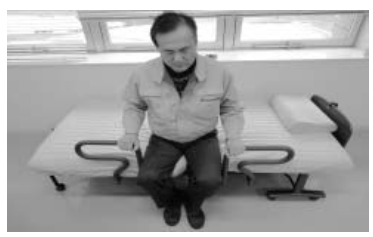

(6) Terminal sitting

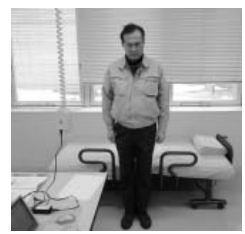

(7) Leaved

Figure 5. Behavior patterns for detection and recognition of bedleaving.

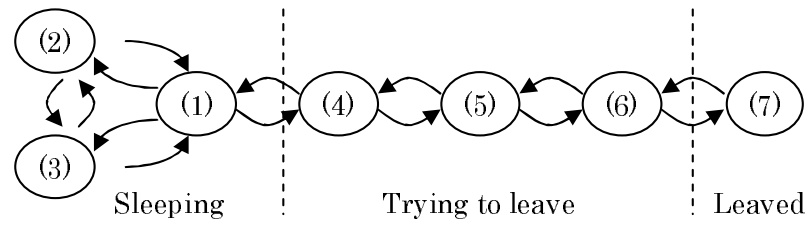

Figure 6. Status transition in respective behavior patterns.

subjects are trying to rise from the bed. We designate this as longitudinal sitting. The second behavior pattern is the status of sitting on the bed to the lateral side. For this position, subjects are trying to move to the terminal of their bed after turning the body from longitudinal sitting. We designate it as lateral sitting. The third behavior pattern is the status of sitting on the terminal of the bed. For this position, subjects are trying to leave the bed. We designate it as terminal sitting. The decision target for this study is to produce seven patterns with the status of complete leaving.

For the recognition of these behavior patterns, we set thresholds of fuzzy values and a definite value to the output voltages from sensors. Our system automatically calculates 
the number of fuzzy values from a histogram. For output related to changes of local parts of the body, a piezoelectric film generates voltage proportionally according to the weight of loading. Moreover, the number of definite values is calculated automatically from the histogram in the case where body weight is used because high voltage is output. The following are the decision criteria combined with fuzzy and definite values of each sensor.

1. Normal sleeping. In this status, a subject is sleeping on the bed normally to the upper side of the body. The decision criteria are that the responses of $\mathrm{S} 1, \mathrm{~S} 2, \mathrm{~S} 3$, or $\mathrm{S} 4$ have intermittent outputs more than fuzzy values, whereas S5 and S6 installed near the terminal of the bed have no response.

2. Rolled right side. In this status, a subject is rolling over to the right side. The decision criteria are the responses of S1 and S3 installed on the right side of the bed, which reach the thresholds of definite values.

3. Rolled left side. In this status, a subject is rolling over to the left side. The decision criteria are the responses of S2 and S4 installed on the left side of the bed, which reach the thresholds of definite values.

4. Longitudinal sitting. In this status, a subject is sitting longitudinally on the bed after rising. The decision criteria are the responses of S3 and S4, installed on the hip of a subject, which reach the thresholds of fuzzy values.

5. Lateral sitting. In this status, a subject is sitting of the lateral of the bed after turning the body from the longitudinal position. The decision criteria are the responses of S3 and S4 and S5 and S6, installed on the terminal of the bed, which reach the thresholds of fuzzy values.

6. Terminal sitting. In this status, a subject is sitting of the terminal of the bed trying to leave the bed. The decision criteria are that the responses of S1, S2, S3, and S4 have no outputs, and that S5 and S6 reach the thresholds of definite values.

7. Leaved. This status is determined as a subject leaving from the bed. Alternatively, a subject is unconscious. The decision criterion is that no sensor has any output.

Figure 6 portrays the status transitions in each pose. In the status of longitudinal sitting, subjects have no direct path for bed-leaving. They will return to normal sleeping. In the status of lateral sitting, subjects will move to leave from their bed because they move to turn the body to the terminal. Therefore, our system must determine the status of the lateral sitting immediately for the prediction of a subject leaving the bed. Moreover, our system rings an alarm immediately if a subject moves their behavior to the status of longitudinal sitting. We consider that our system can protect patients from injury or accidents caused by falling from bed, judging by their status before bed-leaving.

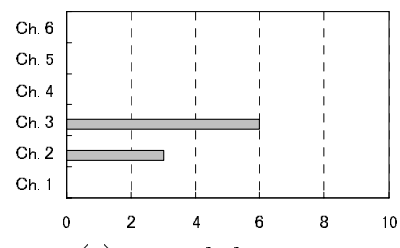

(1) Normal sleeping

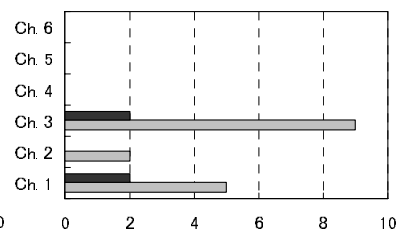

(2) Rolling right side

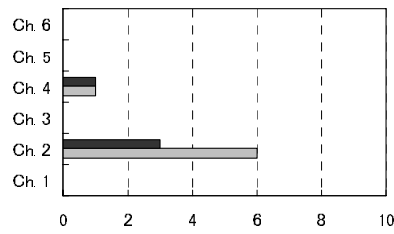

(3) Rolling left side

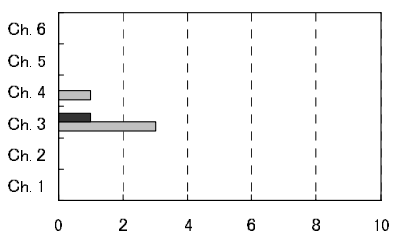

(4) Longitudinal sitting

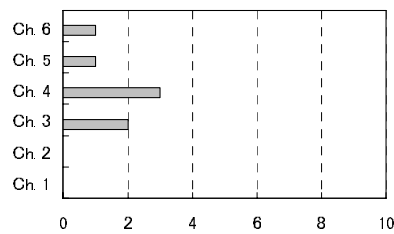

(5) Lateral sitting
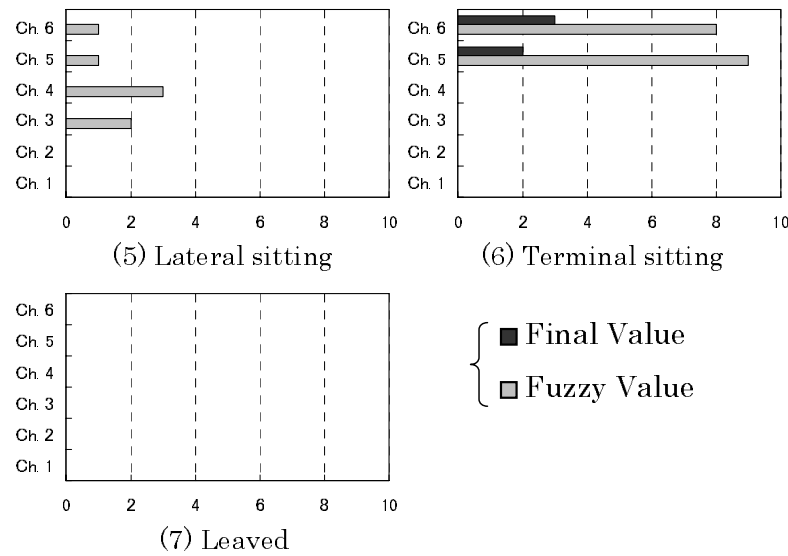

(6) Terminal sitting

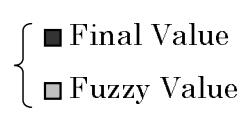

Figure 8. Frequency distributions of fuzzy and definite values in respective behavior patterns.

Table 3. Thresholds of fuzzy values and final values in respective sensors [V].

\begin{tabular}{lcccccc}
\hline Sensor & S1 & S2 & S3 & S4 & S5 & S6 \\
\hline Fuzzy Value & 1.0 & 1.0 & 1.0 & 0.5 & 0.2 & 0.2 \\
Definite Value & 2.0 & 2.0 & 2.0 & 1.0 & 0.5 & 0.5 \\
\hline
\end{tabular}

\section{Experimental results}

We created an experimental environment similar to a clinical site for evaluation of our developed sensor system. The subject is a man in his 50s. We obtained datasets of 10 iterations of stimuli to confirm the reproduction of responses. Figure 5 depicts images related to each status. The person shown in this figure is a subject for this experiment. The subject repeated each status according to the order shown in Fig. 6. Herein, the action period in each status is $20 \mathrm{~s}$. The sampling rate is $50 \mathrm{~Hz}$.

Figure 7 depicts time-series transitions of output voltages in each status. Subsequently, we calculated histograms of fuzzy values and definite values to set thresholds in each channel. Table 3 shows thresholds that we used for this 


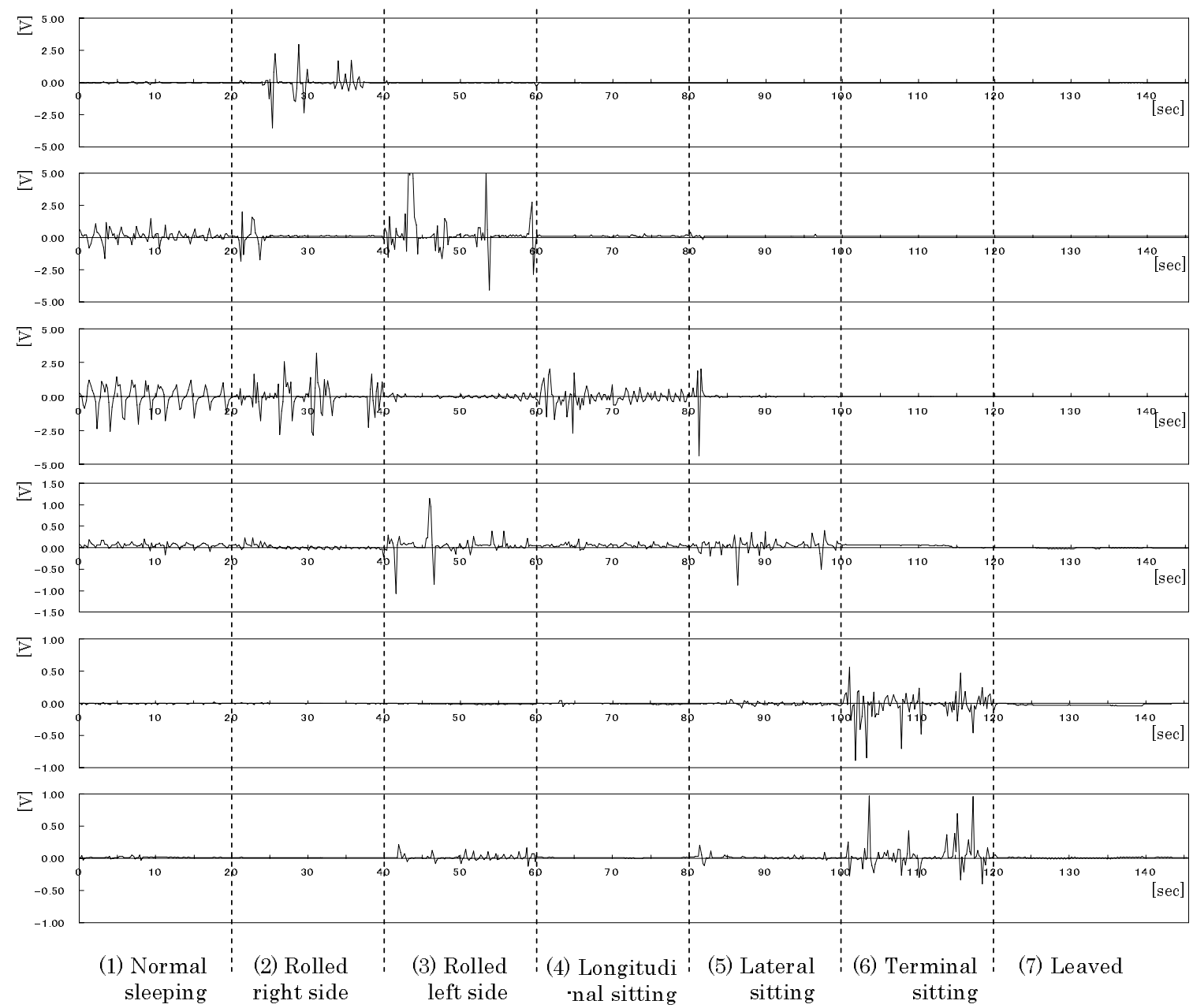

Figure 7. Time-series changes of the sensor output in respective behavior patterns.

experiment. We optimized these thresholds based on results of our preliminary experiments of 10 times. Figure 8 depicts histograms of fuzzy definite values and in each status.

1. For the status of normal sleeping, definite values were not apparent from all sensors; fuzzy values were apparent on S2 and S3. We considered that fuzzy values are generated by slight differences of body pressure according to the sleeping status. Moreover, we considered that the reason for a lack of output from S1 and S4 are influences of the setting of thresholds or the sampling intervals we used for this experiment.

2. For the status of rolled right side, fuzzy and definite values were apparent on S1 and S3, which were installed the left side of the bed. We consider that output voltage reached a definite value because the body weight was concentrated to the lateral side of the body. The fuzzy value can be recognized twice on $\mathrm{S} 2$. We consider that this effect results from body movements.
3. For the status of rolling to the left side, fuzzy and definite values were apparent on $\mathrm{S} 2$ and $\mathrm{S} 4$, which were installed on the right side of the bed. This result shows steady responses because other sensors had no output.

4. For the status of longitudinal sitting, output values were apparent on S3 or S4 installed under the hip of a subject. Other sensors had no output. This result shows the possibility of prediction of a subject to rise and to sit on the longitudinal of the bed.

5. For the status of lateral sitting, fuzzy values were apparent on S1, S2, S3, and S4. The status of this position can be recognized from the output of these four sensors, although definite values were not apparent because of the distribution of the body weight, detected as pressure. This status is the most important to achieve early detection of bed-leaving.

6. For the status of the terminal sitting, the outputs of fuzzy values and definite values were apparent on S5 and S6. 
The fuzzy value and definite value from both sensors were, respectively, eight and two times. This result can be inferred as indicating that the subject tried to execute bed-leaving because the number of outputs of both values are numerous compared with other status. The terminal position immediately before leaving from the bed is a state for a subject required an emergency response to prevent injury or accidents. We consider that our system can detect this position certainly as the most important position among six positions.

7. After leaving the bed, our system can determine bedleaving because no sensor had output values. Herein, the difference between this status and the status of sleeping on the upper side in (1) can be recognized from the distribution of fuzzy values. We consider that this status signals a high emergency state similar to the terminal status. This result means that our method achieved steady detection of bed-leaving.

\section{Discussion}

To detect the status of a subject with long-term and continuous monitoring, we must detect which behaviors in the basic six poses corresponded to a dataset of time-series measurements. From the experimentally obtained result, no fuzzy or definite value on S5 and S6 was observed as a feature in the status of sleeping or rolling of a subject. Moreover, both values showed a distribution according to the status of longitudinal sitting after rising or aiming to bed-leaving from lateral sitting to terminal sitting after turning the body to the lateral side. Therefore, we specifically examined the boundary between attempted leaving and complete leaving for analysis of monitoring data. Consequently, we can realize reduction of the total amount of measured data and protection of false recognition. We regard this is the trigger point that is useful to predict bed-leaving through monitoring.

For prediction of bed-leaving, we obtained a result from Fig. 8 to show a markedly different distribution between the distribution of the histogram of sensors and behavior patterns in the case of the sitting of a subject near the terminal of the bed. For complete leaving, we consider that our results present the possibility of comparative and steady judgments compared with expensive sensors because of a lack of fuzzy response and definite values from all sensors. Compared with the prediction system proposed by Uezono et al. (2010), which used 96 sensors of bending gages, our system can realize detection and prediction of six behavior patterns of bed-leaving only used six sensors: one-sixteenth the number used in their system.

\section{Conclusions}

This paper presented an non-restraining sensor system to predict behaviors of bed-leaving. We developed a prototype of plate-shaped sensors using piezoelectric films. Moreover, we developed a monitoring system consisting of microprocessor boards with wireless modules for collecting data from sensors. According to the seven classified behavior patterns used to predict bed-leaving, we developed decision algorithms from the distribution of histograms. We evaluated our system at an experimental environment constructed in reference to a clinical site. As a result, our system can recognize behavior patterns from the histograms of fuzzy and definite values. Especially, we were able to detect salient behavior features of lateral sitting when trying to leave and terminal status for leaving from the bed compared with other behavior patterns. Moreover, we obtained results enabling us to discern sleeping on the bed with rolling to the left and right side.

For our future work, we must detect behaviors that trigger our system. We must also combine our sensors with other sensors such as acceleration sensors. Moreover, we will obtain steady detection to expand the application range of our method to increase the number of subjects. We would like to apply our system to clinical information network systems such as nurse call networks and electric medical records. Moreover, we would like to apply our system to care facilities or single senior homes for security and safety observation that maintains QOL and privacy together.

\section{References}

Haruyama, K., Tanaka, K., Kobayashi, S., Yasuoka, K., Uchibori, A., and Oka, M.: Development of Getting Up Detection and Report Device using Power Line Communication and Mat-Sensor, Trans. Institute of Electrical Engineers of Japan Part. D, 126, 1507-1513, 2006.

Hirasawa, K., Matsumura, N., Kanemaru, N., and Abe, K.: Falling Accident Prevention System Used for a Clinical Site and Care Facility, Technical Journal of Nippon Telegraph and Telephone, 20, 32-35, 2008.

Imaizumi, K., Iwakami, Y., and Yamashita, K.: Availability of Monitoring System for Supporting Healthcare of Elderly People, Japanese Journal of Applied IT Healthcare, 5, 63-64, 2010.

National Institute of Population and Social Security Research (IPSS), Estimated Population in the Future of Japan, 2012.

Inokuchi, K.: The Labor Shortage in Care Workplace and Employment Intention of the Young, Proc. Kanazawa Univ. Graduate School Human and Socio-Environmental Studies, 15, 69-84, 2008.

Kondo, S., Kamiya, C., Miyamoto, H., Toriyama, Y., Mimura, E., and Tsuchida, F.: Availability of Sensor Mats to Detect Leaving for Protection of Falling Accidents from Bed, Trans. Japanese Association of Rural Medicine, 55, 245, 2006.

Matsuda, H., Yamaguchi, A., and Arakawa, T.: Monitoring System of Living Activities for Elderly People, National Technical Report, 82, 4-8, 2003. 
Measurement Specialties Inc.: Datasheet ofDT Series Elements with Lead Attached, Rev. 1, 2009.

Motegi, M., Matsumura, N., Yamada, T., Muto, N., Kanamaru, N., Shimokura, K., Abe, K., Morita, Y., and Katsunishi, K.: Analyzing Rising Patterns of Patients to Prevent Bed-related Falls (Second Report), Trans. Japan Society for Health Care Management, 12, 25-29, 2011.

National Population Census 2010, Ministry of Internal Affairs and Communications, 2010.

Seki, H. and Hori, Y.: Detection of Abnormal Action Using Image Sequence for Monitoring System of Aged People, Trans. Institute of Electrical Engineers of Japan Part. D, 122, 1-7, 2002.

Shimizu, M., Dugawara, K., Ozaki, F., Hama, Y., Nishimura, M., and Yoshino, H.: Development of Detection System of Getting out of Bed with Ultrasonic Array Sensor (Part 3), Trans. Society of Life Support Technology, 21, 9-16, 2009.
Tanaka, M.: A case example of application of remote monitoring for single living senior persons of biological signal monitoring technology using high sensitive presser sensor made in Finland, Trans. Japanese Telemedical and Telecare Association, 3, 231233, 2007.

Tatsumi, T., Kanemoto, K., and Yagi, N.: How to Use Efficient Tentomushi - Considering the Length of Stride and Height for Protecting False Operation, Proc. Japanese Nursing Association, 38, 144-146, 2007.

Uezono, T., Kubo, A., Nakajo, M., Uekaraseta, A., and Uchida, T.: Application Study of Bed leaving Prediction System, Proc. Conference of Kagoshima Prefectural Industrial Technology Center 2010, 34-35, 2010.

Yamada, R., Takashima, M., Sato, Y., Ito, W., Ito, T., and Asanuma, Y.: Evaluation and Prevention of Inpatient Falls - A study using a classification system based on situational criteria, Proc. Akita Univ. School of Health Sciences, 18, 144-150, 2010. 